\title{
Topological Gaseous Plasmon Polariton in Realistic Plasma
}

\author{
Jeffrey B. Parker®* \\ Lawrence Livermore National Laboratory, Livermore, California 94550, USA \\ J. B. Marston \\ Brown Theoretical Physics Center and Department of Physics, Brown University, Providence, Rhode Island 02912-1843, USA \\ Steven M. Tobias \\ Department of Applied Mathematics, University of Leeds, Leeds, LS2 9JT, United Kingdom \\ Ziyan Zhu० \\ Department of Physics, Harvard University, Cambridge, Massachusetts 02138, USA
}

(Received 4 November 2019; accepted 17 April 2020; published 12 May 2020)

\begin{abstract}
Nontrivial topology in bulk matter has been linked with the existence of topologically protected interfacial states. We show that a gaseous plasmon polariton (GPP), an electromagnetic surface wave existing at the boundary of magnetized plasma and vacuum, has a topological origin that arises from the nontrivial topology of magnetized plasma. Because a gaseous plasma cannot sustain a sharp interface with discontinuous density, one must consider a gradual density falloff with scale length comparable to or longer than the wavelength of the wave. We show that the GPP may be found within a gapped spectrum in presentday laboratory devices, suggesting that platforms are currently available for experimental investigation of topological wave physics in plasmas.
\end{abstract}

DOI: 10.1103/PhysRevLett.124.195001

Edge states arising from topologically nontrivial bulk matter have attracted significant recent attention. For example, topological insulators and the quantum Hall states are now understood as manifestations of topology $[1,2]$, and similar reasoning has been applied to a diverse range of other physical systems [3]. Edge states have garnered intense practical interest due to topological protection and the prospect for robust, undirectional propagation with reduced losses to scattering from defects. In analogy to the systems in the quantum mechanical regime, classical systems, including photonics [4-10], acoustics [11-13], mechanical systems [14,15], as well as continuum fluids [16-19], can exhibit topological quantization as well as edge states between topologically distinct states of matter.

Plasmas support rich wave physics, especially in the presence of a magnetic field, multiple species, kinetic distributions, and inhomogeneity. Analysis of band structure and wave dispersion properties has been a cornerstone in the understanding of plasma waves, leading to important practical applications such as current drive and heating for fusion devices. Yet the topological characterization of plasma band structure, and its consequences for edge states, has not been fully appreciated. One recent work has proposed that the reversed-shear Alfvén eigenmode observed in tokamaks arises from the nontrivial topology associated with magnetic shear and the topological phase transition across a zero-shear layer [20].
Here we consider one of the simplest plasmas, a dilute gas of ions and electrons in a magnetic field. We investigate the topological gaseous plasmon polariton (GPP), an electromagnetic surface wave that arises due to nontrivial topology of a magnetized plasma. The applied magnetic field breaks time-reversal symmetry, and therefore the topology is analogous to that of the integer quantum Hall effect [21] or Kelvin waves [17]. While other surface waves in inhomogeneous or bounded plasmas have been investigated previously [22,23], topological aspects have not been considered. The GPP initially appears from similar mathematical structure as the surface magnetoplasmon polariton occurring at the surface of metals or semiconductors $[24,25]$. However, the internal structures of metals and plasmas are quite different, and quantum corrections are unlikely to play an important role in the gaseous plasma considered here. Another critical difference is that gaseous plasmas, unlike metals and semiconductors, cannot sustain sharp interfaces where the density jumps essentially discontinuously. The spatial variation of the plasma density introduces additional physics such as a changing upper hybrid frequency, and the character of the local dispersion relation may shift across the plasma. The question of whether or not a plasma can support the GPP when the density varies over a length scale larger than a wavelength has not yet been addressed. Potential uses of surface waves like the GPP include surface-mode-sustained plasma discharges [26,27]. 
We consider a plasma with realistic density profile and demonstrate the GPP can be supported, and furthermore we show that parameter regimes in which the GPP is accessible may be attained in currently existing laboratory devices. Our results motivate experiments to probe many of the open issues regarding topological waves in plasmas, such as to what extent they exhibit topological protection, and how nonlinearities affect their behavior.

We adopt the cold-plasma model of a magnetized, stationary plasma, appropriate for light waves when the electron thermal speed is much less than the speed of light. We assume a high-frequency regime and retain only the electron motion, treating ions as an immobile neutralizing background. Electron collisions are neglected for the dilute plasmas considered here because the collision frequency is orders of magnitude smaller than the wave frequency of interest. The linearized equations of motion for an infinite homogeneous plasma are [28]

$$
\begin{aligned}
& \frac{\partial \mathbf{v}}{\partial t}=-\frac{e}{m_{e}}\left(\mathbf{E}+\mathbf{v} \times \mathbf{B}_{0}\right), \\
& \frac{\partial \mathbf{E}}{\partial t}=c^{2} \nabla \times \mathbf{B}+\frac{e n_{e}}{\epsilon_{0}} \mathbf{v}, \\
& \frac{\partial \mathbf{B}}{\partial t}=-\nabla \times \mathbf{E},
\end{aligned}
$$

where $\mathbf{v}$ is the electron fluid velocity, $\mathbf{E}$ the electric field, $\mathbf{B}_{0}=B_{0} \hat{\mathbf{z}}$ the background magnetic field, $\mathbf{B}$ the perturbation magnetic field, $e$ the elementary charge, $n_{e}$ the background electron density, $m_{e}$ the electron mass, $c$ the speed of light, and $\epsilon_{0}$ the permittivity of free space. It is convenient to work in nondimensionalized units in which time is normalized to $\omega_{p}^{-1}$, where $\omega_{p}=\left(n_{e} e^{2} / m_{e} \epsilon_{0}\right)^{1 / 2}$ is the plasma frequency, length to $c / \omega_{p}$, velocity to $e \bar{E} / m_{e} \omega_{p}$, electric field to $\bar{E}$, and magnetic field to $\bar{E} / c$, where $\bar{E}$ is some reference electric field. Then the only parameter is $\sigma=\operatorname{sign}\left(B_{0}\right) \Omega_{e} / \omega_{p}$, where $\Omega_{e}=\left|e B_{0} / m_{e}\right|$ is the electron cyclotron frequency. Upon letting $\partial / \partial t \rightarrow-i \omega$ and $\nabla \rightarrow i \mathbf{k}$, we obtain the eigenvalue equation $H|f\rangle=\omega|f\rangle$, where $|f\rangle=\left[\begin{array}{ll}\mathbf{v} & \mathbf{B}\end{array}\right]$ is a 9-element vector and $H$ is a $9 \times 9$ Hermitian matrix corresponding to the linear operator, which plays the role of an effective Hamiltonian. We work in Cartesian coordinates. The matrix $H$ is written out explicitly in the Supplemental Material [29].

We allow for an arbitrary propagation angle with respect to the magnetic field. We fix $k_{z}$ and consider a parameter space $\mathbf{k}_{\perp}=\left(k_{x}, k_{y}\right)$. This problem is isotropic in the plane perpendicular to the magnetic field. For each $\mathbf{k}_{\perp}$, there are 9 solutions for the eigenvalues $\omega_{n}$, for $n=-4,-3, \ldots, 4$, which we order by ascending frequency, and $\omega_{-n}=-\omega_{n}$. The corresponding eigenfunctions are denoted $|n\rangle$. Except for certain values of $k_{z}$ and $\sigma$, the eigenvalues are nondegenerate. The band structure is shown in Fig. 1.

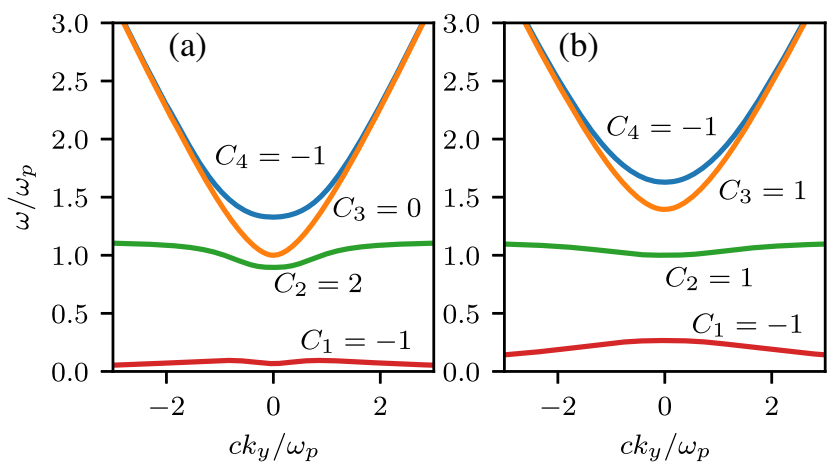

FIG. 1. Spectrum of a magnetized, homogeneous cold plasma as a function of $k_{y}\left(k_{x}\right.$ set to zero, but the system is isotropic in the $x y$ plane), where only electron motion is retained. Here, $\sigma=0.5$. (a) $c k_{z} / \omega_{p}=0.4\left(k_{z}<k_{z}^{*}\right)$. (b) $c k_{z} / \omega_{p}=1.1\left(k_{z}>k_{z}^{*}\right)$. Also shown are the Chern numbers of the positive-frequency bands, computed with the use of the discreteness regularization.

When the eigenvalues are nondegenerate, frequency band $n$ may be characterized by a Chern number $C_{n}=$ $(2 \pi)^{-1} \int d \mathbf{k}_{\perp} F_{n}\left(\mathbf{k}_{\perp}\right)$, where the Berry curvature $F_{n}(\mathbf{k})$ of the band at a given $\mathbf{k}$ is given by

$F_{n}(\mathbf{k})=i \sum_{m \neq n} \frac{\left\langle n\left|\frac{\partial H}{\partial k_{x}}\right| m\right\rangle\left\langle m\left|\frac{\partial H}{\partial k_{y}}\right| n\right\rangle-\left\langle m\left|\frac{\partial H}{\partial k_{x}}\right| n\right\rangle\left\langle n\left|\frac{\partial H}{\partial k_{y}}\right| m\right\rangle}{\left(\omega_{n}-\omega_{m}\right)^{2}}$.

The Chern numbers $C_{1}, C_{3}, C_{4}$ are integer valued, but $C_{2}$ takes noninteger values when using the linear operator in Eq. (1). The issue of a "noninteger Chern number" stems from a lack of insufficient smoothness at small scales of the linear operator $H$, which leads to the inability to compactify the infinite $\mathbf{k}$ plane [16]. If $H$ falls off sufficiently rapidly, one can map the $\mathbf{k}$ plane into the Riemann sphere, which is compact.

An integer $C_{2}$ can be restored through regularization of $H$. Regularization of continuous electromagnetic media based on the notion of material discreteness has been addressed previously [16,30], although other means of compactifying continuum fluids have been discussed $[31,32]$. The plasma ceases to look like a continuous medium at sufficiently small length scales, which the fluid model does not take into account. To model this physical discreteness, the regularization suppresses the plasma response at small scales. In the Fourier representation, the nondimensionalized electron equation of motion is modified to be $\partial_{t} \mathbf{v}=-r(\mathbf{k}) \mathbf{E}-\sigma \mathbf{v} \times \hat{\mathbf{z}}$, where $r$ becomes small at large wave vectors. For instance, we can take $r(\mathbf{k})=\left(1+\left|\mathbf{k}_{\perp}\right|^{2} / k_{c}^{2}\right)^{-1}$ for some cutoff wave number $k_{c}$. This modification functionally alters the plasma frequency to become small at small length scales. To preserve Hermiticity, we also modify the nondimensionalized Ampere-Maxwell equation to be $\partial_{t} \mathbf{E}=\nabla \times \mathbf{B}+r(\mathbf{k}) \mathbf{v}$. 
Using the discreteness regularization, the Chern numbers are integer valued and are independent of the form of $r(\mathbf{k})$ as long as it decays sufficiently rapidly. The regularization removes noninteger contributions from infinite $\mathbf{k}$ while retaining the integer-valued contribution from finite $\mathbf{k}$. A key point is that $k_{c}$ may be taken to be arbitrarily large, such that the physical effect of the regularization at length scales of interest can be made arbitrarily small. Additional details can be found in the Supplemental Material [29].

We consider $k_{z}>0$. Because bands 2 and 3 touch at $k_{z}=k_{z}^{*}$, where $\left(c k_{z}^{*} / \omega_{p}\right)^{2}=\sigma /(1+\sigma)$, there are two distinct regimes: $k_{z}<k_{z}^{*}$ and $k_{z}>k_{z}^{*}$. At $k_{z}<k_{z}^{*}$, the Chern numbers of the positive-frequency bands are $C_{n}=-1,2,0,-1$, for $n=1,2,3,4$, shown in Fig. 1 . The Chern number of the zero-frequency band is 0 , and the Chern numbers of the negative-frequency bands are the negative of their positive-frequency partner. For $k_{z}<k_{z}^{*}$, the band structure smoothly transitions to $k_{z}=0$. At $k_{z}=0$, bands 2 and 4 become $X$ waves, band 3 is the $O$ wave, and band 1 has degenerate frequency $\omega=0$. Different Chern numbers are obtained for $k_{z}>k_{z}^{*}: C_{n}=$ $-1,1,1,-1$ for $n=1,2,3,4$. Hence, multiple plasma bands are topologically nontrivial. If the direction of $\mathbf{B}_{0}$ is reversed, the Chern number also flips sign. The gap Chern number is $\sum_{n=-4}^{1} C_{n}=C_{1}=-1$ for any $k_{z}$.

The gap between the first and second band of the plasma can overlap with a forbidden band in vacuum. The dispersion relation for electromagnetic waves in vacuum is $\omega^{2}=c^{2} k^{2}$. For nonzero $k_{z}$, there is a forbidden region for $\omega^{2}<c^{2} k_{z}^{2}$ in which waves cannot propagate. If the plasma parameters can be engineered such that the band gaps in the plasma and vacuum overlap, bulk-boundary correspondence implies the existence of a undirectional surface mode crossing the gap.

Previous work has considered a wave propagating at the planar, discontinuous interface of a semi-infinite, uniformdensity magnetized plasma and vacuum [33]. However, a sharp interface is not physically realizable for gaseous plasma. The interface width is typically limited by classical or turbulent diffusion processes and may be larger than the length scale of the wave. A notable exception is nonneutral plasma, for which the interface width can be made comparable to the Debye length [34].

To determine whether the GPP can propagate in a realistic plasma, we consider a cylindrical plasma with magnetic field aligned along the $z$ axis. We take into account a density profile that varies smoothly with radius, as shown in Fig. 2(a). For simplicity, we assume a uniform magnetic field. We assume the background plasma has azimuthal symmetry and translational symmetry in $z$. The wave equation for an inhomogeneous cold plasma is simply Eq. (1) with the replacement $n_{e} \rightarrow n_{e}(r)$. For the density profile we use $n_{e}=\frac{1}{2} n_{0}\left(\tanh \left[\left(r_{0}-r\right) / L_{n}\right]+1\right)$, where $L_{n}$ is the length scale over which the density decays.

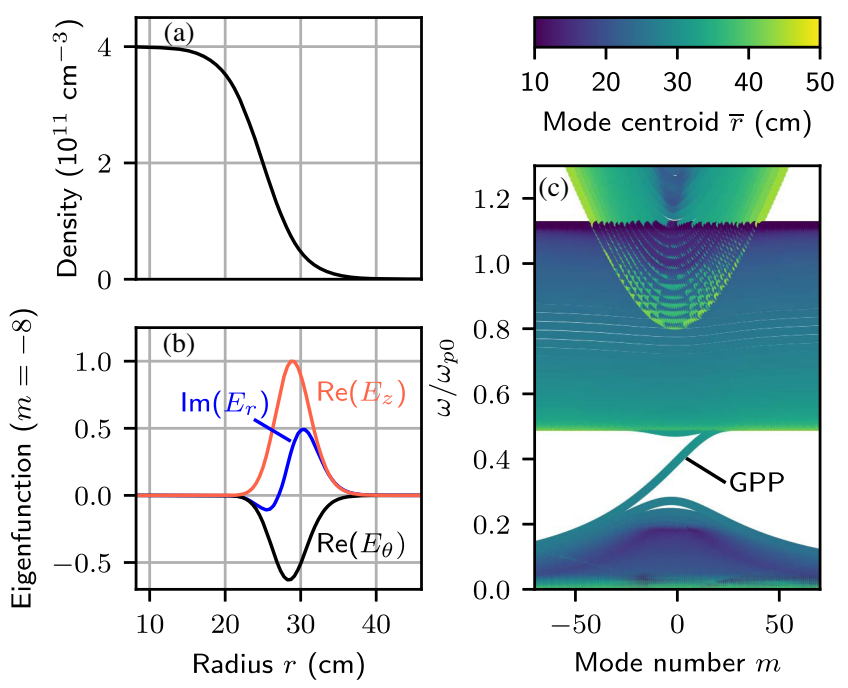

FIG. 2. Spectrum of an inhomogeneous magnetized plasma. Here, $c k_{z} / \omega_{p 0}=0.8$ and $\sigma=0.5$. (a) Plasma density as a function of radius. (b) Nonzero components of GPP electric field at azimuthal mode number $m=-8$. (c) Spectrum as a function of $m$, where color corresponds to the mode centroid of the energy in the electric field. The GPP dispersion relation is indicated and crosses the band gap. There is another mode (not shown) in the numerical solution which is localized to the inner wall and stems from the artificial conducting-wall boundary condition.

We decompose eigenmodes as $f(\mathbf{x}, t)=f(r) e^{i\left(m \theta+k_{z} z-\omega t\right)}$. We solve the radial eigenvalue equation using the spectral code Dedalus [35]. Numerically, we consider a radial domain $[a, b]$ where $a>0$, and for simplicity apply conducting-wall boundary conditions at both $r=a$ and $r=b$. Since the mode of interest is a surface wave localized near $r=r_{0}$, a conducting boundary at $r=a$ can be used even when the physical situation has no inner wall as long as the surface wave has sufficiently small amplitude at $r=a$. It would be preferable to use the more physical boundary condition of no inner wall and requiring only regularity at $r=0$, but we are restricted by our current numerical tools. This more physical geometry could in principle allow the existence of another class of body modes [22]. However, for the specific parameters used here, consideration of the dispersion relation near the plasma center indicates there can be no propagating modes.

The eigenmodes and spectrum are shown for one set of parameters in Figs. 2(b) and 2(c). Here, we take $r_{0}=$ $25 \mathrm{~cm}, L_{n}=5 \mathrm{~cm}, B_{0}=0.1 \mathrm{~T}$, and $n_{0}=4 \times 10^{11} \mathrm{~cm}^{-3}$, which gives $\sigma=\Omega_{e} / \omega_{p 0}=0.5$, where $\omega_{p 0}$ is the plasma frequency computed with $n_{0}$. We take $c k_{z} / \omega_{p 0}=0.8$. These parameters have been chosen because they are accessible to existing laboratory devices. For example, the Large Plasma Device (LAPD) has reported similar magnetic field values and density profiles [36,37]. At these densities and typical electron temperatures $(\sim 10 \mathrm{eV})$, the earlier assumptions justifying the cold plasma model are well satisfied. 


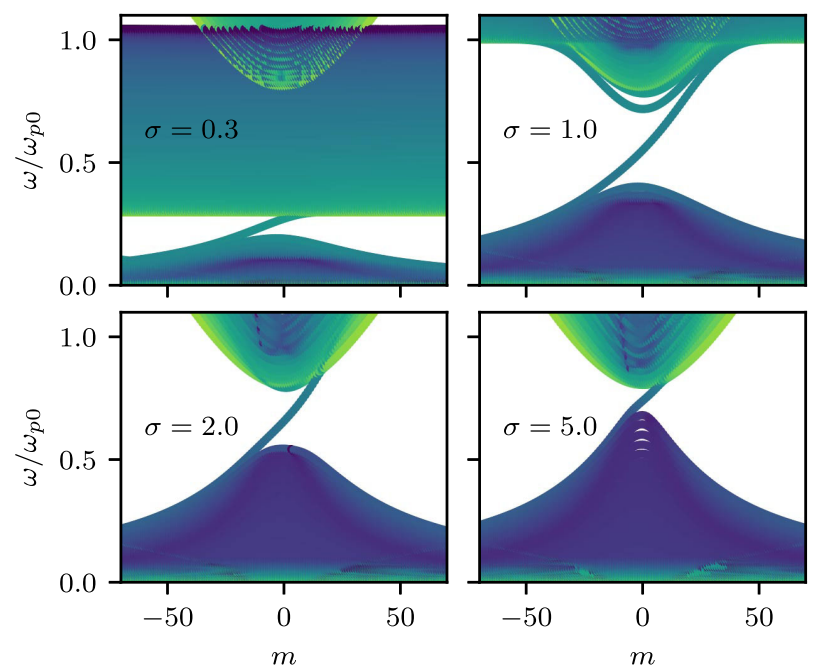

FIG. 3. Spectrum for various magnetic field strengths $\sigma=\left|\Omega_{e}\right| / \omega_{p 0}$. Other parameters and color scale are as in Fig. 2.

In Fig. 2(c), the GPP crosses the band gap $0.3<$ $\omega / \omega_{p 0}<0.5$. The electric-field polarization of the eigenfunction is displayed in Fig. 2(b) for $m=-8$, which shows that the GPP is a surface wave localized to the region between the plasma and vacuum. There are no other waves for the GPP to scatter into at this $k_{z}$.

When $\omega>\Omega_{e}$, upper hybrid modes become accessible. These modes are identifiable as upper hybrid because their frequency is approximately independent of $m$ and the eigenfunctions are localized around the radial location corresponding with the frequency of local upper hybrid oscillations, $\omega^{2}=\omega_{p}^{2}(r)+\Omega_{e}^{2}$. The upper hybrid modes in the low- and intermediate-density region restrict the gapped frequency range and constitute a distinct difference from the spectrum of a plasma and vacuum separated by a sharp interface. If the plasma density is uniform and discontinuously jumps to zero, the only upper hybrid frequency is $\omega_{p 0}^{2}+\Omega_{e}^{2}$.

In a laboratory plasma, the strength of the applied magnetic field is one of the simplest parameters to adjust experimentally and is thus an important control knob. Figure 3 shows how the spectrum varies with the magnetic field strength. For $\Omega_{e}<\omega_{p 0}$, the band gap shrinks because the lowest upper hybrid frequency decreases, and for $\left|\Omega_{e}\right|>\omega_{p 0}$, the band gap shrinks because the top of the lower band rises to meet the bottom of the upper band. Maximizing the size of the band gap, which is achieved for $\Omega_{e} \approx \omega_{p 0}$, will isolate the GPP from other modes and ease its detection.

Our discussion has focused consideration on a single $k_{z}$. The physical system itself is three dimensional, which unlike the two-dimensional case, is not expected to have topologically suppressed scattering [38]. However, the existence of the surface wave is still protected topologically against small perturbations. Furthermore, magnetized plasmas tend to be much more uniform along the magnetic field than perpendicular to it. Physically, this is because charged particles stream freely along a magnetic field line but are tied to the field line in the perpendicular direction by the cyclotron motion. As a result, plasma nonuniformities along a magnetic field are smoothed out very quickly, and there would be very low spectral power in modes with $k_{z} / k_{\perp} \sim 1$. This separation of scales has been observed to hold in the LAPD, with flutelike drift-Alfvén perturbation modes that have very small $k_{z}$ [39] and fluctuate slowly (tens of $\mathrm{kHz})$ compared to the GPP $(\sim \mathrm{GHz})$. Therefore, to a first approximation, the system is translationally invariant and $k_{z}$ will be conserved.

In summary, we have shown that the gaseous plasmon polariton, which arises from the nontrivial topology of waves in a bulk magnetized plasma, can exist at the plasmavacuum interface with a realistic, gradual plasma density falloff. The density scale length can be comparable to the wavelength. For certain choices of plasma density and magnetic field, the wave propagates in a gapped frequency range and thus may be able to serve as a protected probe of plasma in tokamaks and other plasma devices. We have shown that such parameter regimes are achievable in presentday cylindrical plasma devices, such as the Large Plasma Device at the Basic Plasma Science Facility. Laboratory experiments to confirm the existence of this topological edge mode are therefore in reach. Properties of the wave that can be predicted and compared with measurements include the frequency, dispersion relation, radial localization, and polarization. Such experiments could confirm the first controlled observation of a wave of topological origin in a gaseous plasma.

We acknowledge useful discussions with Troy Carter, Bart Van Compernolle, George Morales, Hong Qin, and Shreekrishna Tripathi. J. B. P.'s work was performed under the auspices of the U.S. Department of Energy by Lawrence Livermore National Laboratory under Contract No. DE-AC52-07NA27344. J. B. P. and J. B. M. would like to acknowledge the workshop Vorticity in the Universe held at the Aspen Center for Physics in the summer of 2017 and supported by National Science Foundation Grant No. PHY1607611, which played an important role in bringing about this work. S. M. T. is supported by the European Research Council (ERC) under the European Union Horizon 2020 research and innovation program (Grant Agreement No. D5S-DLV-786780). Z.Z. is supported by the STC Center for Integrated Quantum Materials, NSF Grant No. DMR1231319.

*jbparker3@wisc.edu

[1] M. Z. Hasan and C. L. Kane, Colloquium: Topological insulators, Rev. Mod. Phys. 82, 3045 (2010).

[2] X.-L. Qi and S.-C. Zhang, Topological insulators and superconductors, Rev. Mod. Phys. 83, 1057 (2011). 
[3] I. Martin, Y. M. Blanter, and A. F. Morpurgo, Topological Confinement in Bilayer Graphene, Phys. Rev. Lett. 100, 036804 (2008).

[4] Z. Wang, Y. Chong, J. D. Joannopoulos, and M. Soljacić, Observation of unidirectional backscattering-immune topological electromagnetic states Nature (London) 461, 772 (2009).

[5] L. Feng, M. Ayache, J. Huang, Y.-L. Xu, M.-H. Lu, Y.-F. Chen, Y. Fainman, and A. Scherer, Nonreciprocal light propagation in a silicon photonic circuit, Science 333, 729 (2011).

[6] Y. Plotnik, M. C. Rechtsman, D. Song, M. Heinrich, J. M. Zeuner, S. Nolte, Y. Lumer, N. Malkova, J. Xu, A. Szameit, Z. Chen, and M. Segev, Observation of unconventional edge states in "photonic graphene", Nat. Mater. 13, 57 (2014).

[7] S. A. Skirlo, L. Lu, and M. Soljacić, Multimode One-Way Waveguides of Large Chern Numbers, Phys. Rev. Lett. 113, 113904 (2014).

[8] L. Lu, J. D. Joannopoulos, and M. Soljacić, Topological photonics, Nat. Photonics 8, 821 (2014).

[9] S. A. Skirlo, L. Lu, Y. Igarashi, Q. Yan, J. Joannopoulos, and M. Soljacić, Experimental Observation of Large Chern Numbers in Photonic Crystals, Phys. Rev. Lett. 115, 253901 (2015).

[10] S. A. H. Gangaraj and F. Monticone, Topologicallyprotected one-way leaky waves in nonreciprocal plasmonic structures, J. Phys. Condens. Matter 30, 104002 (2018).

[11] V. Peano, C. Brendel, M. Schmidt, and F. Marquardt, Topological Phases of Sound and Light, Phys. Rev. X 5, 031011 (2015).

[12] Z. Yang, F. Gao, X. Shi, X. Lin, Z. Gao, Y. Chong, and B. Zhang, Topological Acoustics, Phys. Rev. Lett. 114, 114301 (2015).

[13] C. He, X. Ni, H. Ge, X.-C. Sun, Y.-B. Chen, M.-H. Lu, X.-P. Liu, and Y.-F. Chen, Acoustic topological insulator and robust one-way sound transport, Nat. Phys. 12, 1124 (2016).

[14] L. M. Nash, D. Kleckner, A. Read, V. Vitelli, A. M. Turner, and W. T. M. Irvine, Topological mechanics of gyroscopic metamaterials, Proc. Natl. Acad. Sci. U.S.A. 112, 14495 (2015).

[15] S. D. Huber, Topological mechanics, Nat. Phys. 12, 621 (2016).

[16] M. G. Silveirinha, Chern invariants for continuous media, Phys. Rev. B 92, 125153 (2015).

[17] P. Delplace, J. B. Marston, and A. Venaille, Topological origin of equatorial waves, Science 358, 1075 (2017).

[18] S. Shankar, M. J. Bowick, and M. C. Marchetti, Topological Sound and Flocking on Curved Surfaces, Phys. Rev. X 7, 031039 (2017).

[19] M. Perrot, P. Delplace, and A. Venaille, Topological transition in stratified fluids, Nat. Phys. 15, 781 (2019).

[20] J. B. Parker, J. W. Burby, J. B. Marston, and S. M. Tobias, Nontrivial topology in the continuous spectrum of a magnetized plasma, arXiv:1909.07910.

[21] D. J. Thouless, M. Kohmoto, M. P. Nightingale, and M. den Nijs, Quantized Hall Conductance in a Two-Dimensional Periodic Potential, Phys. Rev. Lett. 49, 405 (1982).
[22] A. W. Trivelpiece and R. W. Gould, Space charge waves in cylindrical plasma columns, J. Appl. Phys. 30, 1784 (1959).

[23] B. N. Breizman and A. V. Arefiev, Radially Localized Helicon Modes in Nonuniform Plasma, Phys. Rev. Lett. 84, 3863 (2000).

[24] J. J. Brion, R. F. Wallis, A. Hartstein, and E. Burstein, Theory of Surface Magnetoplasmons in Semiconductors, Phys. Rev. Lett. 28, 1455 (1972).

[25] H. Bin, Z. Ying, and W. Q. Jie, Surface magneto plasmons and their applications in the infrared frequencies, Nanophotonics 4, 383 (2015).

[26] C. M. Ferreira, Theory of a plasma column sustained by a surface wave, J. Phys. D 14, 1811 (1981).

[27] Y. M. Aliev, H. Schlüter, and A. Shivarova, Guided-WaveProduced Plasmas (Springer, New York, 2000).

[28] T. Stix, Waves in Plasmas (American Institute of Physics, College Park, MD, 1992).

[29] See Supplemental Material at http://link.aps.org/ supplemental/10.1103/PhysRevLett.124.195001 for the effective Hamiltonian matrix and additional details regarding the calculation of the Chern number.

[30] G. W. Hanson, S. A. H. Gangaraj, and A. Nemilentsau, Notes on photonic topological insulators and scattering-protected edge states: A brief introduction, arXiv:1602.02425.

[31] C. Tauber, P. Delplace, and A. Venaille, A bulk-interface correspondence for equatorial waves, J. Fluid Mech. 868, 10.1017/jfm.2019.233 (2019).

[32] A. Souslov, K. Dasbiswas, M. Fruchart, S. Vaikuntanathan, and V. Vitelli, Topological Waves in Fluids with Odd Viscosity, Phys. Rev. Lett. 122, 128001 (2019).

[33] B. Yang, M. Lawrence, W. Gao, Q. Guo, and S. Zhang, Oneway helical electromagnetic wave propagation supported by magnetized plasma, Sci. Rep. 6, 21461 (2016).

[34] J. R. Danielson and C. M. Surko, Radial compression and torque-balanced steady states of single-component plasmas in Penning-Malmberg traps, Phys. Plasmas 13, 055706 (2006).

[35] K. J. Burns, G. M. Vasil, J. S. Oishi, D. Lecoanet, and B. P. Brown, Dedalus: A flexible framework for numerical simulations with spectral methods, Phys. Rev. Research 2, 023068 (2020).

[36] W. Gekelman, P. Pribyl, Z. Lucky, M. Drandell, D. Leneman, J. Maggs, S. Vincena, B. Van Compernolle, S. K. P. Tripathi, G. Morales, T. A. Carter, Y. Wang, and T. DeHaas, The upgraded large plasma device, a machine for studying frontier basic plasma physics, Rev. Sci. Instrum. 87, 025105 (2016).

[37] J. E. Maggs, T. A. Carter, and R. J. Taylor, Transition from Bohm to classical diffusion due to edge rotation of a cylindrical plasma, Phys. Plasmas 14, 052507 (2007).

[38] Y. Ando, Topological insulator materials, J. Phys. Soc. Jpn. 82, 102001 (2013).

[39] J. R. Peñano, G. J. Morales, and J. E. Maggs, Drift-Alfvén fluctuations associated with a narrow pressure striation, Phys. Plasmas 7, 144 (2000). 